\title{
Mechanisms of secondary resistance to tyrosine kinase inhibitors in gastrointestinal stromal tumours (Review)
}

\author{
ALESSANDRA MALEDDU, MARIA A. PANTALEO, MARGHERITA NANNINI, MONICA DI BATTISTA, \\ MARISTELLA SAPONARA, CRISTIAN LOLLI and GUIDO BIASCO \\ ${ }^{1}$ Institute of Hematology and Medical Oncology 'L\&A Seràgnoli’, Sant'Orsola-Malpighi Hospital, ${ }^{2}$ Interdepartmental \\ Centre for Cancer Research 'G. Prodi', University of Bologna, I-40138 Bologna, Italy
}

Received January 30, 2009; Accepted March 11, 2009

DOI: $10.3892 /$ or_00000361

\begin{abstract}
Treatment of patients affected by advanced or inoperable GIST was revolutionized by the use of the tyrosine kinase inhibitors. Despite the fact that most patients have a good durable response of disease, they develop a resistance to treatments after a median time of 24 months. The acquired resistance is an emerging aspect in medical oncology especially in the era of target therapies. The aim of this review is to report all known mechanisms of secondary resistance to tyrosine kinase inhibitors and to highlight their clinical implications. In general, they may be divided in mechanisms related to the acquisition of new molecular abnormalities associated to KIT and PDGFRA receptor signalling pathway, such as the loss of KIT expression, the genomic amplification of KIT, the activation of an alternative downstream signalling pathways such as AKT/mTOR and the acquisition of new receptor mutations, and other mechanisms different to KIT/PDGFRA receptors. Future research perspectives on target therapy and early resistance evaluation are also discussed.
\end{abstract}

\section{Contents}

1. Introduction

2. Evidence of KIT and PDGFRA mutations

3. Mechanisms of secondary resistance

4. Tyrosine kinase receptor-related mechanisms

5. Other mechanisms of secondary resistance

6. Clinical implications and perspectives

7. Conclusions

Correspondence to: Dr Alessandra Maleddu, Institute of Hematology and Medical Oncology 'L\&A Seràgnoli', Sant'Orsola-Malpighi Hospital, University of Bologna, Via Massarenti 9, I-40138 Bologna, Italy

E-mail: alessandramaleddu@gmail.com

Key words: gastrointestinal stromal tumours, KIT, PDGFRA, secondary resistance, mutations

\section{Introduction}

Gastrointestinal stromal tumours (GISTs) arise from the interstitial cells of Cajal (ICC). They are the most common mesenchymal tumours of the gastrointestinal tract, harbouring mainly in the stomach. The oncogenic event that leads to GIST development is a gain-of-function KIT or PDGFRA gene mutation that results in the constitutive ligand-independent activation of receptors and of their downstream signalling with the loss of control of the cellular cycle, cell proliferation and resistance to apoptosis (1). As well known, GISTs are poorly responsive to radiotherapy and conventional chemotherapy (2). No effective medical treatment was available until 2001 when imatinib mesylate $\left(\right.$ Gleevec ${ }^{\circledR}$ ) was successfully introduced to treat advanced unresectable GISTs (3). The development of tyrosine kinase inhibitors and their wide use in clinical practice has dramatically changed the natural history of GISTs and at moment imatinib mesylate represents the standard medical treatment for advanced unresectable disease. In fact, about $75-90 \%$ of patients with advanced GISTs treated with imatinib have a clinical benefit (stable disease or partial/complete response) (3). Despite such a high response rate, there are subsets of tumours that are primarily resistant to imatinib. As widely demonstrated, the response to tyrosine kinase inhibitors is strictly correlated to KIT and PDGFRA mutational status (4). Heinrich et al reported that patients with exon 11 KIT mutations have a response rate of $83.5 \%$, compared to $48 \%$ for patients with exon 9 mutations (4). Moreover, patients with exon 11 mutations have a longer event-free survival and median survival than patients with exon 9 mutations $(4,5)$. In particular, specific KIT exon 11 point mutation V560G is associated with increased susceptibility to imatinib inhibition of kinase activity (6). Patients with exon 18 PDGRFA D842V point mutation are not usually sensitive to imatinib $(7,8)$. Finally, wild-type KIT/PDGFRA GISTs are also more resistant to imatinib, but the percentage of response or stable disease is variable according to several studies $(4,5,9)$.

Also the patients who have a good response to imatinib quite invariably develop disease progression. Several mechanisms of secondary resistance have been proposed among which the predominant one seems to be a reactivation of the receptors due to a new acquisition of a point of mutation in KIT or PDGFRA conferring imatinib resistance (10). 


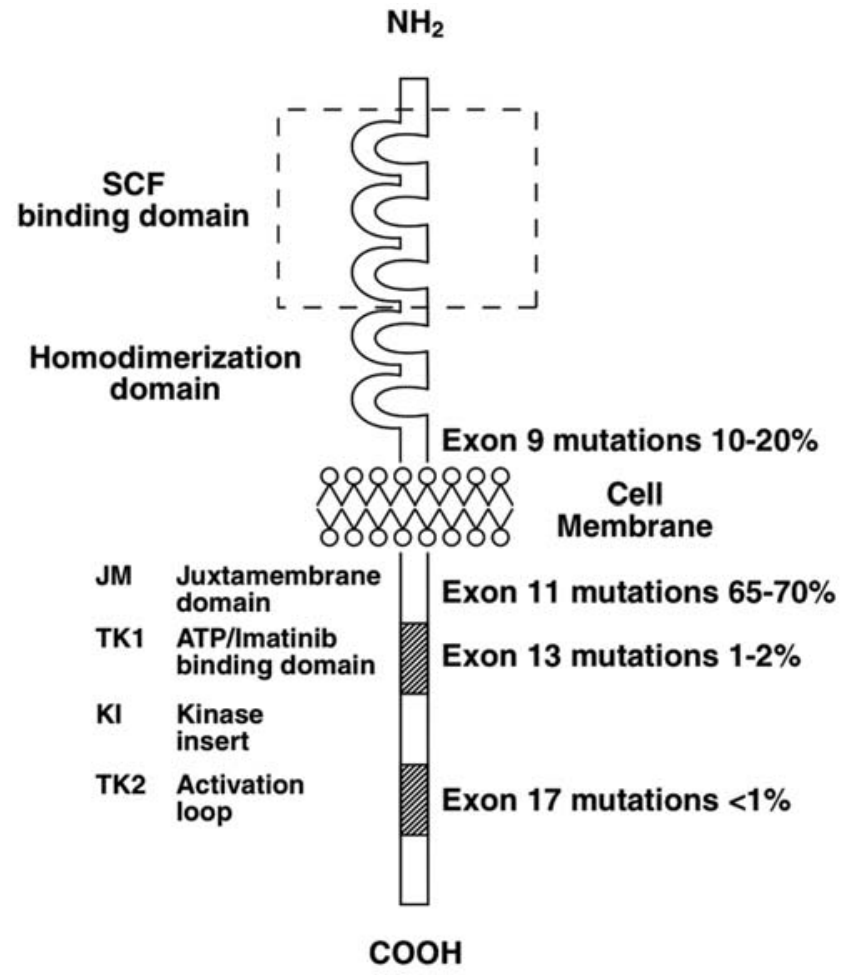

Figure 1. Structure and mutations of KIT receptor.

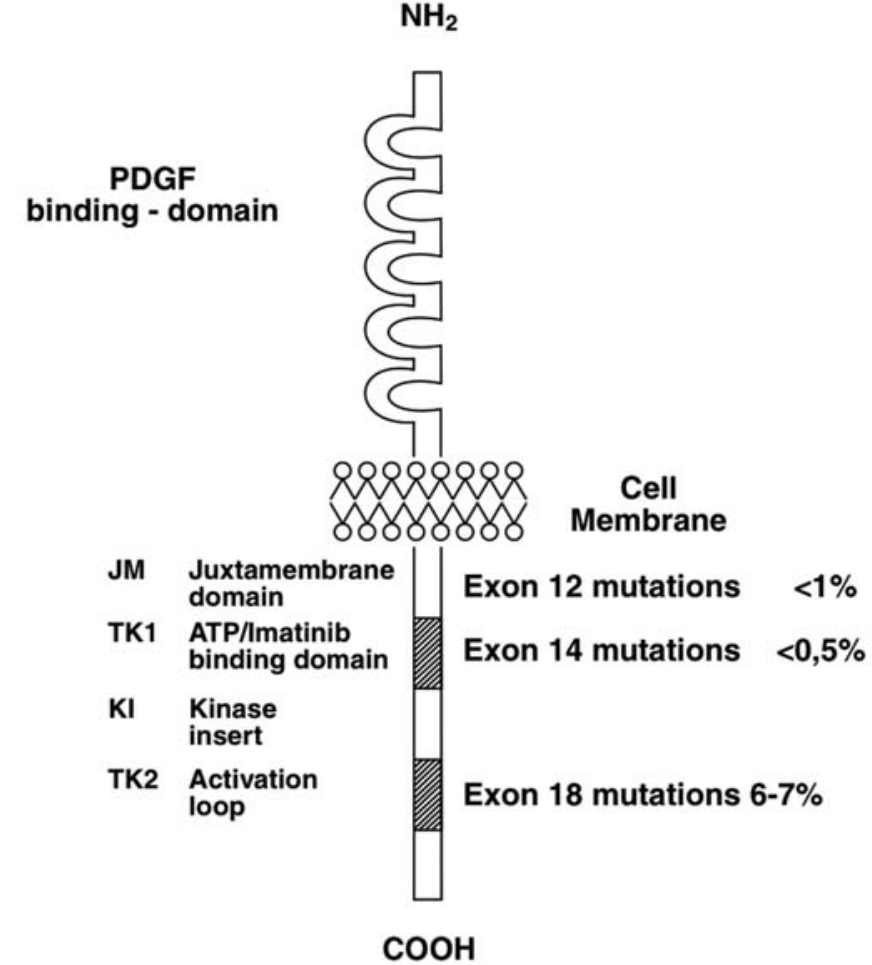

Figure 2. Structure and mutations PDGFr $\alpha$ receptor.
The aim of this review is to report all known mechanisms of secondary resistance of GISTs to tyrosine kinase inhibitors, to highlight their clinical implications and to discuss future research perspectives on target therapy and early response evaluation.

\section{Evidence of KIT and PDGFRA mutations}

KIT and PDGFRA genes are both located in chromosome $4 q 12$. They encode for transmembrane receptors that belong to the type III tyrosine kinase family whose natural ligand are stem cell factors (SCF) and plateled derived growth factors (PDGF). The structure of the receptor is similar for KIT and PDGFRA, made of an extracellular domain with five immunoglobulin-like domains, a transmembrane domain and an intracellular domain which contains two tyrosine kinase domains: the first one with an adenosine triphosphate (ATP) binding region and the second one with a phosphotransferase region (activation loop). The activation of receptors normally occurs with the ligand binding, and consequently with the dimerization of the receptor, then autophosphorylation of the tyrosine kinase domain and finally, the activation of substrates of KIT/PDGFRA such as PI3K/Akt, Ras/MAPK and JAK/STAT which promotes cell cycle activation, cell proliferation, and apoptosis inhibition $(11,12)$. Several gain of function mutations of KIT and PDGFRA in different exons have been reported (Figs. 1 and 2) $(13,14)$. Hirota et al in 1998 demonstrated for the first time the key role of KIT activating mutations in GISTs pathogenesis. They examined six GIST samples, found KIT activating mutation in five, all located in the juxta-membrane domain (exon 11) within Lys550 and Val 560, responsible for constitutive activation of the KIT receptor $(1,15)$. Afterwards several groups confirmed these data and other KIT exon 11 mutations have been identified such as an in-frame deletion involving codons encoding amino acid residues from 550 to 579 , in particular the 557 codon, and less frequently points of mutation (mainly in 557, 559, 560 and 576 codons), duplications, insertions and complex mutations $(4,5,13,14,16,17)$. Mutation of the juxta-membrane domain brings KIT constitutive activation and was observed mainly in tumours with gastric localization $(14,16)$. Mutations in other exons of KIT receptor were reported (16-23). Lux et al identified an insertion of six nucleotides in exon 9 which leads to an amino acidic duplication of Ala-Tyr in the extracellular domain of the receptor (18). This mutation is normally found in about $11 \%$ of GISTs, however its frequency is significantly associated with large size tumours and arising in the small bowel $(14,16,19,20,23)$.

In a small number of GIST some exon 13 mutations, among which the $\mathrm{K} 642 \mathrm{E}$ point mutation is the most common, harbouring in the tyrosine kinase site $(18,19,22)$. Even less frequently, mutations affecting the activation loop (exon 17) are involved in the pathogenesis of GISTs. The N822K and $\mathrm{N} 822 \mathrm{H}$ have been identified in sporadic GISTs, whereas D820Y has been observed in familiar GISTs $(21,22,24)$.

Evidence of PDGFRA mutations in KIT-wild-type (WT) GIST was demonstrated for the first time by Heinrich et al in 2003. Using GIST478 cell line they observed that phosphoPDGFRA (activated PDGFRA) was highly expressed in this cell line and that phosphoPDGFRA and phosphoKIT expression are mutually exclusive. Moreover, in cells with high expression of phosphoPDGFRA, they identified a missense D $842 \mathrm{~V}$ mutation and an in-frame deletion of amino acid residues $842-845$ of the exon 18 which encodes for the PDGFRA activation loop (7). Other sites of mutation are further identified in exon 18 (D842Y, deletion 842 to 

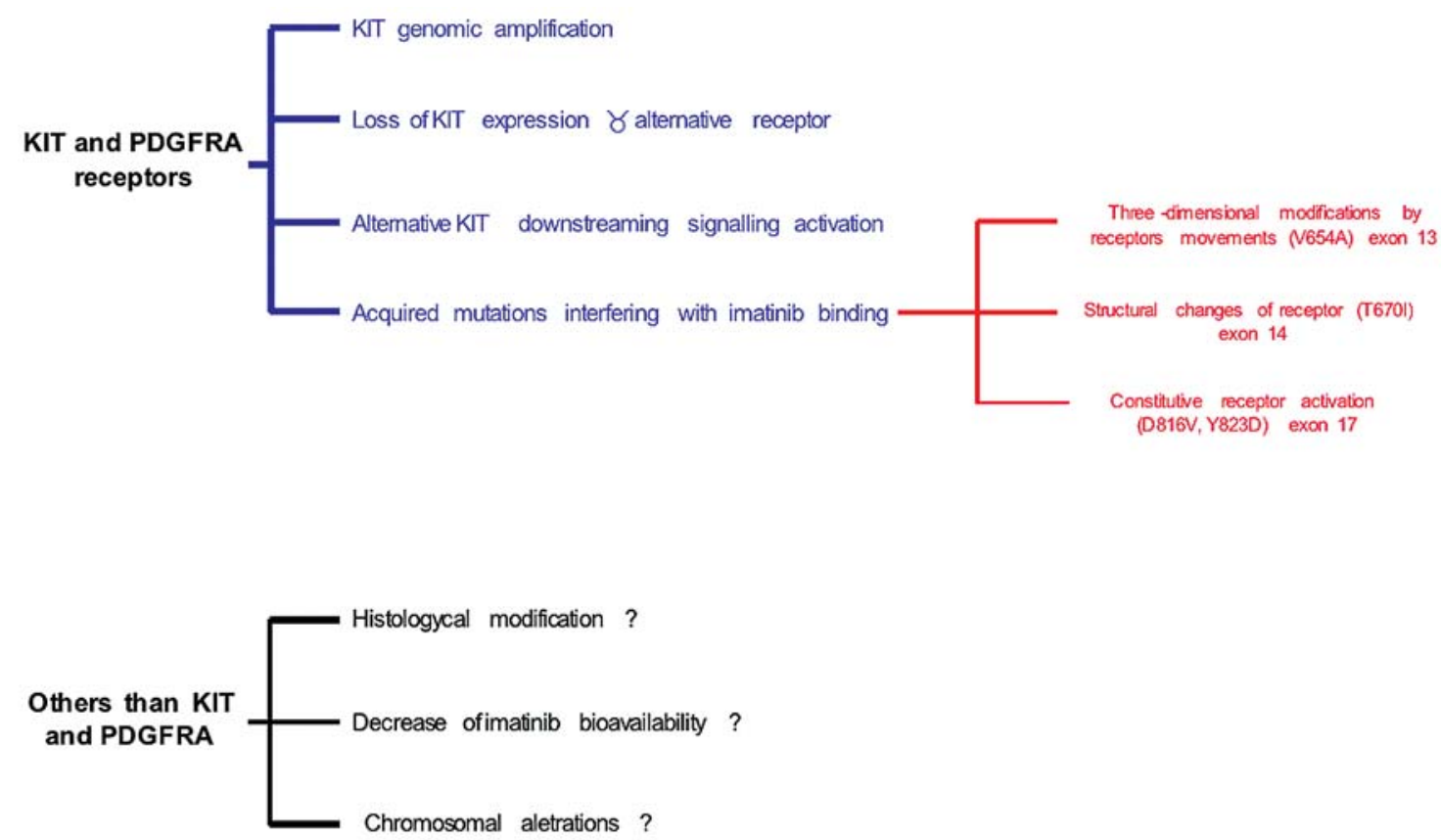

Figure 3. Main known mechanisms of secondary resistance to tyrosine kinase inhibitors in GISTs.

845-delDIMH842-845-, delRD841-842KN), exon 12 (delSPDGHE566-571-R, del560-564) exon 14 (N659K) (25).

As well known, mutational status of KIT and PDGFRA affects prognosis and response to tyrosine kinase inhibitors, and it may confer primary or secondary resistance $(4,26)$.

\section{Mechanisms of secondary resistance}

The mechanisms of secondary resistance to tyrosine kinase inhibitors have been widely investigated (Fig. 3). The demonstrated mechanisms are related to abnormalities of KIT and PDGFRA receptors (structure and function). Other mechanisms are supposed such as chromosomal alterations, pharmacokinetic variables or tumour differentiation.

\section{Tyrosine kinase receptor-related mechanisms}

Disease progression under imatinib treatment may occur after a median time to progression of about 24 months. Knowledge on chronic myeloid leukemia (CML) has been helpful to understand drug resistance mechanisms in GIST. In fact chronic myeloid leukaemia develops resistance to imatinib through several cellular mechanisms, one of which is the acquisition of new BCR/ABL gene mutations (27). Fletcher et al identified four different mechanisms of imatinib secondary resistance associated to KIT and PDGFRA receptor in GISTs: i) the activation of an alternative downstream signalling pathways such as AKT/mTOR; ii) the activation of an alternative tyrosine kinase receptor and loss of KIT expression; iii) the genomic amplification of KIT; iv) the acquisition of new KIT/PDGFRA mutations. At present the latter is considered the most important and the most frequent one (Table I) (10).

Chen et al studied a series of 12 GISTs with primary mutations in exons 9 and 11 which developed imatinib resistance after an initial response (28). Mutational analysis of six resistant lesions revealed in all of them the presence of the V654A mutation in the tyrosine kinase domain I, encoded by exon 13 , later recognized as the most commonly acquired mutation (28-31). Subsequent studies allowed to identify other acquired points of mutation, especially in KIT exon 14 (resulting in the T670I substitution) and in exon 17 (resulting in the Y823D, D716N, D816G and D820E /Y substitution, N822K) (32-34). Antonescu et al performed mutational analysis of 31 tumour nodules from patients treated with imatinib classified as 'non-resistant', 'primary-resistant' and 'acquired-resistant' (35). When possible more than one nodule of the same patient was analyzed. Results showed the absence of new mutations in non-resistant tumours. Instead, in 7 of 15 progressing patients secondary KIT mutations were found. The most common site of secondary mutations was exon 17 with substitution N822K, D820K and Y823D, whereas other already known points of mutations were identified in exon 13 (V654A) and in exon 14 (T760I). In a patient who underwent recurrence resection, two different mutations were found: the first one was located in exon 13 (V654A) and the second one was found in the exon 17 (N822K). In 3 patients the secondary mutations were present only in one, but not in all nodules. Successively, the occurrence of more than one acquired KIT mutation in the same patient was described (36-38). Waldermann et al studied 32 patients with progressive disease under imatinib treatment and found secondary mutations in 14 patients: 10 patients developed only 1 new mutation, and 4, 3,2 mutations were found in 3 different patients. No more than one mutation was detected in the same lesion. Moreover, primary mutations can be detected in all tumour samples before and after imatinib whereas, in consequence of clonal evolution, secondary mutations were expressed exclusively in progressing lesions (36). The theory of clonal evolution of some nodules 
Table I. Secondary resistance mutations to tyrosine kinase inhibitors in GISTs.

\begin{tabular}{|c|c|c|c|c|c|c|}
\hline Author & Year & $\begin{array}{c}\text { No. of } \\
\text { patients }\end{array}$ & Primary mutation & $\begin{array}{c}\text { Sequenced } \\
\text { samples }\end{array}$ & Secondary mutation & Refs. \\
\hline Chen LL & 2004 & 5 & 1 exon 9-4 exon 11 & 6 & KIT exon 13 (V654A) & (28) \\
\hline Tamborini E & 2004 & 1 & Exon 11 & 1 & KIT exon 14 (T670I) & (32) \\
\hline Wakai T & 2004 & 1 & Exon 11 & 1 & KIT exon 17 (Y823D) & (33) \\
\hline Antonescu CR & 2005 & 15 & $\begin{array}{l}3 \text { exon 9-11 exon 11-1 KIT/ } \\
\text { PDGFRA WT }\end{array}$ & 16 & $\begin{array}{l}\text { KIT exon } 13(\mathrm{~V} 654 \mathrm{~A}) \\
\text { KIT exon } 14(\mathrm{~T} 670 \mathrm{I}) \\
\text { KIT exon } 17 \text { (N822K, D820Y, Y823D) }\end{array}$ & (35) \\
\hline Debiec-Rychter M & 2005 & 26 & $\begin{array}{l}6 \text { exon 9-19 exon 11-1 KIT/ } \\
\text { PDGFRA WT }\end{array}$ & 26 & $\begin{array}{l}\text { KIT exon } 13 \text { (V654A) } \\
\text { KIT exon } 14 \text { (T670I) } \\
\text { KIT exon } 17 \text { (D716N, D820E, D820Y, } \\
\text { N822K, D816G) PDGFRA (D842V) }\end{array}$ & (34) \\
\hline Waldermann E & 2005 & 32 & $\begin{array}{l}7 \text { exon 9-22 exon 11-3 KIT/ } \\
\text { PDGFRA WT }\end{array}$ & 104 & $\begin{array}{l}\text { KIT exon } 13 \text { (V654A) } \\
\text { KIT exon } 14 \text { (T670I, T670E, S709F) } \\
\text { KIT exon } 17 \text { (D816E, D820E, D820G, } \\
\text { D820Y, N822K, Y823D) }\end{array}$ & (36) \\
\hline Tamborini E & 2005 & 1 & Exon 11 & 1 & KIT exon 13 (V654A) & (29) \\
\hline Grimpen F & 2005 & 2 & Exon 11 & 2 & KIT exon 17 D816H, Arg-Asp 815-816 del & $(64)$ \\
\hline Heinrich MC & 2006 & 33 & $\begin{array}{l}3 \text { exon } 9-25 \text { exon } 11-1 \text { exon } 13- \\
1 \text { exon } 13 \text { +exon } 17-1 \text { exon } 11+ \\
\text { exon } 13-1 \text { PDGFRAV561D-2 } \\
\text { KIT/D816H, D820A, D820G, } \\
\text { PDGFRA WT }\end{array}$ & 54 & $\begin{array}{l}\text { KIT exon } 13(\mathrm{~V} 654 \mathrm{~A}) \\
\text { KIT exon } 14(\mathrm{~T} 670 \mathrm{I}) \\
\text { KIT exon } 17(\mathrm{C} 809 \mathrm{G}, \mathrm{Y} 823 \mathrm{D}, \mathrm{N} 822 \mathrm{~K})\end{array}$ & (9) \\
\hline Bertucci F & 2006 & 1 & Exon 9 & 1 & KIT exon 13 (V654A) & $(31)$ \\
\hline Utsunomyia $\mathrm{T}$ & 2007 & 1 & Exon 11 & 1 & KIT exon 13 (V654A) & $(30)$ \\
\hline Lim KH & 2008 & 12 & $\begin{array}{l}3 \text { exon 9-8 exon 11-1 KIT/ } \\
\text { PDGFRA WT }\end{array}$ & ND & $\begin{array}{l}\text { KIT exon } 11 \text { (V559A) } \\
\text { KIT exon } 13 \text { (V654A, insertion } 643 \\
\text { nucleotide A frameshift) KIT exon } 17 \\
\text { (D820G, D820Y, N822K) PDGFRA } \\
\text { exon } 14 \text { (H687Y) PDGFRA } \\
\text { exon } 18(\mathrm{~V} 824 \mathrm{~V})\end{array}$ & (38) \\
\hline Nishida $\mathrm{T}$ & 2008 & 25 & 3 exon $9-22$ exon 11 & 45 & $\begin{array}{l}\text { KIT exon } 13 \text { (V654A, N822K, Y823D) } \\
\text { KIT exon } 14 \text { (T670I) } \\
\text { KIT exon } 17 \text { (K786N, C809G, D816H, } \\
\text { D816E, D820V, N822Y, N822K, N822D, } \\
\text { Y823D, A829P) }\end{array}$ & (37) \\
\hline Liegl B & 2008 & 5 & 1 exon 9-3 exon $11-1$ exon 13 & 16 & $\begin{array}{l}\text { KIT exon } 11 \text { (V559A) } \\
\text { KIT exon } 13 \text { (V654A) } \\
\text { KIT exon } 17 \text { (D820G, D820Y, Y823D) } \\
\text { KIT exon } 18 \text { (A829P) }\end{array}$ & (39) \\
\hline
\end{tabular}

resistant to imatinib was also described by Desai et al (6). They demonstrated that in 48 patients with progressive disease, 23 patients developed a resistant nodule (defined as a new enhancing nodule within a pre-existing mass) detectable about five months before the objective progression (as increment of tumour size). In $80 \%$ of these patients, secondary mutations were detected. The clonal evolution of resistant nodules detected by CT-scan or FDG-PET may be considered a pattern of progressing disease (6).

In a recent paper Liegl et al highlighted the wide spectrum of resistance mechanisms to tyrosine kinase inhibitors with different mutations found in different samples taken from the 
same lesion or in different lesions from the same patient (39). They analyzed 53 metastases from 14 patients after imatinib or sunitinib treatment failure. Primary tumours were not only GIST with classical features, KIT positive tumours with KIT exon 9, 11, 13 mutations, but also KIT negative, with unusual morphology and KIT/PDGFRA WT-GISTs. Secondary KIT mutations were found in 9 of 11 GIST with KIT primary mutation. In 6 of 9 patients were found 2-5 different mutations in separate metastases and in 3 of 9 patients two mutations were found in one or more tumour samples. Recurrent point of mutations were five (V654A, D820G, N822K ( $\rightarrow$ A and $\mathrm{T} \rightarrow \mathrm{G}), \mathrm{Y} 823 \mathrm{D})$, located in the KIT tyrosine kinase domain and in the ATP activation loop. Interestingly, in KIT/PDGFRA WT-GISTs and in those with an unusual morphology no secondary mutations were found. Wide heterogeneity within and between metastases in the same patient is a crucial emerging GIST feature. Different resistance mechanisms such as genomic amplifications detected by Fluorescence In Situ Hybridization (FISH) was also investigated and found in 1 patient.

In general, frequency of secondary acquired mutations under imatinib therapy was about $50 \%$ in those tumours with primary KIT exon 9 or 11 mutations. Secondary mutations are single substitutions and occur in different exons but on the same allele of primary mutation. Similar to chronic myeloid leukemia, acquired imatinib resistant mutations affects the tyrosine kinase domain and the activation loop, respectively encoded by exons 13,14 and 17 (14,28,32-35,39). The most common secondary mutation is the V654A, mainly in GIST which harbour an exon 11 primary mutation (9). In general, secondary mutations were detected only in progressive nodules and not in non-progressive ones $(35,40)$. Patients with WT-GIST do not develop mutations (39).

PDGFRA secondary mutations are rare. The D842V was identified in 1 patient with primary mutation V561D, which is notably associated with imatinib resistance (9).

Secondary mutations may also occur in a distinct receptor to those affected by the primary mutation but it is uncommon. The first case was described by Debiec-Rychter et al, a patient who had a KIT G565R primary mutation became imatinib resistant through a D842V PDGFRA point mutation (34). The second case was described by Lim et al, a patient who had a primary KIT mutation in exon 9 developed a secondary mutation in PDGFRA exon 14 (H687Y) (38).

From a biological point of view, acquired mutations confer drug resistance through conformational changes or constitutive activation of receptor (notably imatinib binds the inactive form of tyrosine kinase) $(41,42)$. A missense mutation that leads to an amino acidic substitution may create some movement and may modify the three-dimensional structure of receptor interfering with imatinib binding, such the V654A mutations $(43,44)$. A conformational change of the ATP/ imatinib binding pocket occurs in the case of T670I mutation, which corresponds to the T315I mutation of BCR/ABR in CML where the threonine makes a critical hydrogen bond with imatinib, and the loss confers resistance to imatinib (43-45). Finally, acquired mutations may confer imatinib resistance by constitutive stabilization of kinase activity which precludes the imatinib binding as the D816V and the Y823D mutation $(35,43)$. Notably, an activating mutation is normally found in no more than one KIT exon in GIST specimens before imatinib treatment, whereas more than one different KIT mutations is frequently found in progressing GIST lesions.

In conclusion, there is a relatively small set of secondary mutations, nevertheless they generate high intralesional and interlesional variability. The obvious consequence is the development of a multi-drug resistant disease or also, depending on the point of view, the development of different tumours in the same patient.

\section{Other mechanisms of secondary resistance}

As well known, a wide spectrum of cellular abnormalities are found in progressing GISTs under imatinib therapy. Some of them may be responsible for treatment resistance in alternative to secondary alterations of KIT and PDGFRA receptors signalling pathway. In order to understand the complex biology of progressing lesions, a more comprehensive molecular study is necessary. Debiec-Rychter et al performed a histological examination on primary and progressing lesions that revealed loss of histological spindle features, loss of KIT expression and acquisition of epithelioid aspect in progressing ones from 2 different patients (34). More recently Liegl et al observed the presence of rhabdomyosarcomatous differentiation areas adjacent to classic GIST areas in metastatic lesions from 5 patients progressing to tyrosine kinase treatments (in 2 cases the primary GISTs have a spindle cell morphology and in the remaining 3 cases an epithelioid morphology) (46). Primary KIT mutations were still detectable in rhabdomyoblastic areas of the progressing nodules, but no evidence of secondary mutations was found (46).

Other mechanisms of secondary imatinib resistance have been described in vitro studies such as the overexpression of the oncogenic kinase receptor AXL, as 'kinase switch', or the modifications of cellular transporter mechanisms of kinase inhibitors $(47,48)$. However, until now scarce data are available in GIST on the contrary to CML. In fact, some observations on the role of drug transporters on resistance to imatinib have already been reported in patients with chronic myeloid leukemia (CML) (49-51). The human organic cation transporter 1 (hOCT1) is an active transporter of imatinib into cells and its expression or its genetic polymorphisms may influence the intracellular level of imatinib and consequently its effectiveness (52). Attention to this matter should also be paid in GISTs. Finally, chromosomal alterations in GISTs have already been demonstrated by the comparative genomic hybridisation (CGH) such as the loss of chromosome $14 q$, chromosome $22 q$, and chromosome $1 p(53,54)$. The significance of these macroscopic alterations in terms of treatments resistance has not been described.

In addition to the cellular and molecular profile of disease, large variations of imatinib through plasma levels have been reported in GIST patients (55; Demetri GD, et al, Gastrointestinal Cancer Symposium: abs. 3, 2008). The decrease of imatinib bioavailability during chronic therapies should be considered as further interesting possible mechanism of resistance. Recently Demetri et al (60) studied the imatinib pharmacokinetic and pharmacodynamic profiles in advanced GIST patients to detect possible correlations between the 
imatinib through plasma concentrations and clinical outcome. They observed that patients with the lowest imatinib serum levels had lowest overall response rate and shortest time to progression.

The patient's compliance to the drug administration, the pharmacokinetic variability in the tyrosine kinase inhibitor metabolism and transporter enzymes and other aspects should be considered as possible variants influencing the treatment responses and patients outcome.

\section{Clinical implications and perspectives}

Most of the secondary resistance mechanisms are related to tumour genotyping and in particular, multiple and co-existing mutations or the presence of other abnormalities associated with treatment resistance was reported. The observation that within the tumour mass may coexist synchronously resistant and sensitive foci leads clinicians to review both the medical and surgical therapeutic approach of GISTs (56-58). Regarding medical treatment, at present patients progressing on first line imatinib treatment have the possibility of imatinib dose escalation or treatment with sunitinib $(59,60)$. New compounds are under investigation such as nilotinib, dasatinib, the heat shock protein 90 inhibitor IPI-504 or the mTOR inhibitor silorimus $(48,61,62)$. Some of them are investigated in patients resistant to both imatinib and sunitinib as usually required by clinical research steps. Nevertheless advanced GIST patients are often widely pre-treated, consequently their diseases have acquired new molecular abnormalities which are different in comparison to previously untreated settings. Clinicians should ask themselves if it makes sense to test new drugs firstly in widely pre-treated patients, whose resistant diseases are biologically different from primary ones. If we can expect a 'new' disease over time, the role of second, third line and further line therapy should be revised. The results from clinical trials in patients with imatinib- or sunitinib-resistant GISTs may not be useful to plan first line clinical trials. However, a crucial objective is to overcome the multiple mechanism resistance typical of advanced disease, and one possible way may be the combination of multiple tyrosine kinase inhibitors, the investigation of a new role for chemotherapy and its combination with tyrosine kinase inhibitors.

The detection of these new developed biological abnormalities is difficult for various reasons. First of all, invasive procedures are required to collect samples. Moreover, the information derived from ex vivo analysis is related to small tumour specimens and it is not representative of the global disease especially in the metastatic setting where foci of resistance and foci of response were previously well described. To overcome these difficulties some future challenges should be considered. A non-invasive global analysis of the molecular background of the primary tumour and the metastases may be more useful for the clinical practice. The molecular imaging approach with functional technologies such as positron emission tomography (PET) using conventional but also novel tracers specifically directed to molecular abnormalities could provide information on in vivo distribution of biological markers and could improve the selection of patients before therapies (63). The correlation between functional data obtained by imaging technology and molecular background of disease may help the clinicians in the management of GIST patients (64). Moreover, this approach may be applied serially in different steps of the disease. As already reported, 18F-fluorodeoxyglucose (FDG) uptake strongly decreases after imatinib, even $24 \mathrm{~h}$ after the start of the administration, and showed good potential in the early prediction of tumour response or primary resistance by evaluating the variations of glucose metabolism (65-67). FDG-PET may also detect foci of secondary resistance by the re-emergence of glycolytic activity early probably due to the acquisition of new mutation within mass FDG negative after imatinib (68). However, FDG-PET evaluates only the glucose metabolism and may not always be useful for monitoring the molecular inhibition to therapy. This aspect in addition to the possibility of a heterogenous response to TK inhibitors in metastatic disease suggests and encourages the development and the study of novel PET radiotracers (69-72). 18-Fluorodeoxythymidine (FLT) has already been demonstrated to be a marker for monitoring antiproliferative drug in oncology because it provides information directly on the cell proliferation inhibition and not through the reduction of glucose transporter enzymes as for FDG (69).

PET tracers specifically targeting the therapeutic molecules have been developed. Kil et al have recently synthesized a PET tracer labeling imatinib with $11 \mathrm{C}$ which is directed to activated KIT (70). This tracer may be useful in monitoring the focal drug sensitiveness and assessing the focal drug resistance as well. In addition, the PET imaging of vascular endothelial growth factor receptor (VEGFr) may provide information on the effect of sunitinib, even its antiangiogenic activity in GIST is still controversial. Wang et al have reported a preclinical study on a tracer targeting VEGFr-2, the 64-Cu-DOTA-VEGF(121) and 64-Cu-DOTAVEGF(DEE), showing the tumour accumulation in 4T1 xenograft models expressing VEGFr-2 using small animal PET (71). The future translation of these PET tracers in the clinical setting seems promising but is still far, and further studies and more efforts are still needed.

Another challenge is a more comprehensive genome analysis, not only of KIT and PDGFRA oriented, that may provide global information about molecular background and new therapeutic targets. In the recent study of Liegl et al the multiple and co-existing mutations or the presence of other abnormalities associated with treatment resistance were identified thanks to the combination of different molecular methodologies like D-HPLC (Denaturing High Performance Liquid Chromatography) and AS-PCR (Allele Specific Polymerase Chain Reaction) and FISH (39). This finding suggests that multiple-assay approach is required in order to obtain more detailed tumour genotyping. Moreover, in the case of wild-type tumours the absence of kinase domain mutations suggest the importance of investigating other genetic changes. A whole genome analysis may be added to the standard analysis of mutational and amplification status of the receptors. For example, the array-technologies that have the advantage of screening the global genome through a high-resolution analysis, may provide both the gene expression profiling underlying to a specific genotype and the copynumber alterations (73-75). The array analysis with single- 
nucleotide polymorphisms array (SNPs array) was recently introduced for characterizing the whole cancer genome and has demonstrated a great potential for identifying still unknown genes involved in cancer (76). Obviously, the power of using different assays, genotype-specific and wide genome, needs to be investigated in a large series.

The combination of the information coming from molecular imaging technologies and molecular biology (array and gene oriented analyses) may contribute to identify the specific areas of resistance and the myriad of molecular alterations other than KIT and PDGFRA that may have a role on drug responsiveness (77). However, great efforts are needed to realize this approach.

\section{Conclusions}

The acquisition of new biological abnormalities may occur during the natural history of metastatic disease especially in long-time and heavily treated patients and it may be responsible for the secondary resistance. In GISTs, the most common mechanism is related to the alterations of the receptor signalling pathways, in particular the acquisition of new mutations. The secondary mutations may often be multiple and intra- and interlesions heterogeneous especially in the metastatic disease. In the future, combination therapies and the use of conventional and high laboratory assays together with innovative molecular imaging technology may improve the treatment of every single patient affected by a disease with a kaleidoscopic molecular alteration.

\section{References}

1. Hirota S, Isozaki K, Moriyama Y, et al: Gain of function mutations of c-kit in human gastrointestinal stromal tumors. Science 279: 577-580, 1998.

2. Plaat BE, Hollema H, Molenaar WM, et al: Soft tissue leiomyosarcomas and malignant gastrointestinal stromal tumors: differences in clinical outcome and expression of multidrug resistance proteins. J Clin Oncol 18: 3211-3220, 2000.

3. Demetri GD, von Mehren M, Blanke CD, et al: Efficacy and safety of imatinib mesylate in advanced gastrointestinal stromal tumors. N Engl J Med 347: 472-480, 2002.

4. Heinrich MC, Corless CL, Demetri GD, et al: Kinase mutations and imatinib response in patients with metastatic gastrointestinal stromal tumors. J Clin Oncol 21: 4342-4349, 2003.

5. Debiec-Rychter M, Dumez H, Judson I, et al: Use of c-KIT/ PDGFRA mutational analysis to predict the clinical response to imatinib in patients with advanced gastrointestinal stromal tumours entered on phase I and II studies of the EORTC Soft Tissue and Bone Sarcoma Group. Eur J Cancer 40: 689-695, 2004.

6. Desai J, Shankar S, Heinrich MC, et al: Clonal evolution of resistance to imatinib in patients with metastatic gastrointestinal stromal tumors. Clin Cancer Res 13: 5398-5405, 2007.

7. Heinrich MC, Corless CL, Duensing A, et al: PDGFRA activating mutations in gastrointestinal stromal tumors. Science 299: 708-710, 2003 .

8. Corless CL, Schroeder A, Griffith D, et al: PDGFRA mutations in gastrointestinal stromal tumors: frequency, spectrum and in vitro sensitivity to imatinib. J Clin Oncol 23: 5357-5364, 2005.

9. Heinrich MC, Corless CL, Blanke CD, et al: Molecular correlates of imatinib resistance in gastrointestinal stromal tumors. J Clin Oncol 24: 4764-4774, 2006.

10. Fletcher JA, Corless CL, Dimitrijevic S, et al: Mechanisms of resistance to imatinib mesylate (IM) in advanced gastroinestinal stromal tumor (GIST). Proc ASCO 22: abs. 3275, 2003.

11. Isozaki K and Hirota S: Gain-of-function mutations of receptor tyrosine kinases in gastrointestinal stromal tumors. Curr Genomics 7: 469-475, 2006.
12. Kitamura $\mathrm{Y}$ and Hirota S: Kit as a human oncogenic tyrosine kinase. Cell Mol Life Sci 61: 2924-2931, 2004.

13. Lasota $\mathrm{J}$ and Miettinen M: Clinical significance of oncogenic KIT and PDGFRA mutations in gastrointestinal stromal tumours. Histopathology 53: 245-266, 2008.

14. Corless CL, Fletcher JA and Heinrich MC: Biology of gastrointestinal stromal tumors. J Clin Oncol 22: 3813-3825, 2004.

15. Nakahara M, Isozaki K, Hirota S, et al: A novel gain-of-function mutation of c-KIT gene in gatsrointestinal stromal tumors. Gastroenterology 115: 1090-1095, 1998.

16. Antonescu CR, Sommer G, Sarran L, et al: Association of KIT exon mutations with non-gastric primary site and aggressive behaviour: KIT mutation analysis and clinical correlates of 120 gastrointestinal stromal tumors. Clin Cancer Res 9: 3329-3337, 2003.

17. Cho S, Kitadai Y, Yoshida S, et al: Deletion of the KIT gene is associated with liver metastases and poor prognosis in patients with gastroinetstinal stromal tumors in the stomach. Int J Oncol 28: 1361-1367, 2006.

18. Lux ML, Rubin BP, Biase TL, et al: KIT extracellular and kinase domain mutations in gastrointestinal stromal tumors. Am J Pathol 156: 791-795, 2000.

19. Lasota J, Wozniak A, Sarlomo-Rikala M, et al: Mutations in exons 9 and 13 of KIT gene are rare events in gastrointestinal stromal tumors. Am J Pathol 157: 1091-1095, 2000.

20. Hirota S, Nishida T, Isozaki K, et al: Gain-of-function mutation at the extracellular domain of KIT in gastrointestinal stromal tumours. J Pathol 193: 505-510, 2001.

21. Rubin BP, Singer S, Tsao C, et al: KIT activation is a ubiquitous feature of gastrointestinal stromal tumors. Cancer Res 61: 8118-8121, 2001

22. Kinoshita K, Isozaki K, Hirota S, et al: c-kit gene mutation at exon 17 or 13 is very rare in sporadic gastrointestinal stromal tumors. J Gastroenterol Hepatol 18: 147-151, 2003.

23. Sakurai S, Oguni S, Hironaka M, et al: Mutations in c-KIT gene exons 9 and 13 in gastrointestinal stromal tumors among Japanese. Jpn J Cancer Res 92: 494-498, 2001

24. Hirota S, Nishida T, Isozaki K, et al: Familial gastrointestinal stromal tumors associated with dysphagia and novel type germline mutation of KIT gene. Gastroenterology 122: 1493-1499, 2002.

25. Medeiros F, Corless CL, Duensing A, et al: KIT-negative gastrointestinal stromal tumors. Am J Surg Pathol 28: 889-894, 2004.

26. Heinrich MC, Maki RG, Corless CL, et al: Primary and secondary kinase genotypes correlate with the biological and clinical activity of sunitinib in imatinib-resistant gastrointestinal stromal tumor. J Clin Oncol 33: 5352-5359, 2008.

27. Gorre ME, Mohammed M, Ellwood K, et al: Clinical resistance to STI-571 cancer therapy caused by BCR-ABL gene mutation or amplification. Science 293: 886-880, 2001.

28. Chen LL, Trent JC, Wu EF, et al: A missense mutation in KIT kinase domain 1 correlates with imatinib resistance in gastrointestinal stromal tumors. Cancer Res 64: 5913-5919, 2004.

29. Tamborini E, Gabanti E, Lagonigro MS, et al: KIT/Val654Ala receptor detected in one imatinib-resistant GIST patient. Cancer Res 65: 1115, 2005.

30. Utsunomyia T, Okamoto M, Yano S, et al: Secondary c-kit mutation in a recurrent gastrointestinal stromal tumor under long-term treatment with imatinib mesylate: report of a case. Surg Today 38: 65-67, 2008.

31. Bertucci F, Goncalves A, Monges G, et al: Acquired resistance to imatinib and secondary KIT exon 13 mutation in gastrointestinal stromal tumour. Oncol Rep 16: 97-101, 2006.

32. Tamborini E, Bonadiman L, Greco A, et al: A new mutation in the KIT ATP pocket causes acquired resistance to imatinib in a gastrointestinal stromal tumor patient. Gastroenterology 127: 294-299, 2004.

33. Wakai T, Kanda T, Hirota S, et al: Late resistance to imatinib therapy in a metastatic gastrointestinal stromal tumour is associated with a second KIT mutation. Br J Cancer 90: 2059-2061, 2004.

34. Debiec-Rychter M, Cools J, Dumez H, et al: Mechanisms of resistance to imatinib mesylate in gastrointestinal stromal tumors and activity of the PKC412 inhibitor against imatinib-resistant mutants. Gastroenterology 128: 270-279, 2005.

35. Antonescu CR, Besmer P, Guo T, et al: Acquired resistance to imatinib in gastrointestinal stromal tumors occurs through secondary gene mutation. Clin Cancer Res 11: 4182-4190, 2005 . 
36. Waldermann E, Merkelbach-Bruse S, Pauls K, et al: Polyclonal evolution of multiple secondary KIT mutations in gastrointestinal stromal tumors under treatment with imatinib mesylate. Clin Cancer Res 12: 1743-1749, 2006.

37. Nishida T, Kanda T, Nishitani A, et al: Secondary mutations in the kinase domain of the KIT gene are predominant in imatinibresistant gastrointestinal stromal tumor. Cancer Sci 99: 799-804, 2008.

38. Lim KH, Huang M-J, Chen L-T, et al: Molecular analysis of secondary kinase mutations in imatinib-resistant gastrointestinal stromal tumors. Med Oncol 25: 207-213, 2008.

39. Liegl B, Kepten I, Le C, et al: Heterogeneity of kinase inhibitor resistance mechanisms in GIST. J Pathol 216: 64-74, 2008.

40. Al-Batran S-E, Hartmann JT, Heidel F, et al: Focal progression in patients with gastrointestinal stromal tumors after initial response to imatinib mesylate: a three-center-based study of 38 patients. Gastric Cancer 10: 145-152, 2007.

41. Schindler T, Bornmann W, Pellicena P, et al: Structural mechanism for STI-571 inhibition of abelson tyrosine kinase. Science 289: 1938-1942, 2000.

42. Mol CD, Dougan DR, Schneider TR, et al: Structural basis for the autoinhibition and STI-571 inhibition of c-kit tyrosine kinase. J Biol Chem 279: 31655-31663, 2004.

43. Roberts KG, Odell AF, Byrnes EM, et al: Resistance to c-KIT kinase inhibitors conferred by V654A mutation. Mol Cancer Ther 6: 1159-1166, 2007.

44. Tamborini E, Pricl S, Negri T, et al: Functional analyses and molecular modeling of two c-KIT mutations responsible for imatinib secondary resistance in GIST patients. Oncogene 25 6140-6146, 2006.

45. Bohmer FD, Karagyozov L, Uecker A, et al: A single aminoacid exchange inverts susceptibility of related receptor tyrosine kinases for the ATP site inhibitor STI-571. J Biol Chem 278 5148-5155, 2003.

46. Liegl B, Hornick JL, Antonescu CR, Corless CL and Fletcher CD: Rhabdomyosarcomatous differentiation in gastrointestinal stromal tumors after tyrosine kinase inhibitor therapy: a novel form of tumor progression. Am J Surg Pathol (In press).

47. Mahadevan D, Cooke L, Riley C, et al: A novel tyrosine kinase switch is a mechanism of imatinib resistance in gastrointestinal stromal tumors. Oncogene 26: 3909-3919, 2007.

48. Prenen H, Guetens G, De Boek G, et al: Cellular uptake of the tyrosine kinase inhibitors imatinib and AMN 107 in gastrointestinal stromal tumor cell lines. Pharmacology 77: 11-16, 2006.

49. Thomas J, Wang L, Clark RE and Pirmohamed M: Active transport of imatinib into and out of cells: implications for drug resistance. Blood 104: 3739-3745, 2004.

50. Crossman LC, Druker BJ, Deininger WN, et al: hOCT1 and resistance to imatinib. Blood 106: 1133-1134, 2005.

51. Wang L, Giannoudis A, Lane S, et al: Expression of the uptake drug transporter hOCT1 is an important clinical determinant of the response to imatinib in chronic myeloid leukemia. Clin Pharmacol Ther 83: 258-264, 2008.

52. Schinkler AH and Jonker JW: Polymorphisms affecting function of the human organic cation transporter hOCT1(SLC22A1) what are the consequences? Pharmacogenetics 12: 589-590, 2002.

53. Wozniak A, Sciot R, Guillou L, et al: Array CGH analysis in primary gastrointestinal stromal tumours: cytogenetic profile correlates with anatomic site and tumor aggressiveness, irrespective of mutational status. Genes Chromosomes Cancer 46: 261-276, 2007.

54. Assamaki R, Sarlomo-Rikala M, Lopez-Guerrero JA, et al: Array comparative genomic hybridization analysis of chromosomal imbalance and their target genes in gastrointestinal stromal tumours. Genes Chromosomes Cancer 46: 564-576, 2007.

55. Bui BN, Italiano A, Miranova A, Bouchet S and Molimard M: Trough imatinib plasma levels in patients treated for advanced gastrointestinal stromal tumors evidence of large interpatient variations under treatment with standard doses. Proc ASCO 26: abs. 10564, 2008.

56. Gronchi A, Fiore M, Miselli F, et al: Surgery of residual disease following molecular-targeted therapy with imatinib mesylate in advanced/metastatic GIST. Ann Surg 245: 341-346, 2007.

57. Raut CP, Posner M, Desai J, et al: Surgical management of advanced gastrointestinal stromal tumors after treatment with targeted systemic therapy using kinase inhibitors. J Clin Oncol 24: $2325-2331,2006$.
58. DeMatteo RP, Maki RG, Singer S, et al: Results of tyrosine kinase inhibitor therapy followed by surgical resection for metastatic gastrointestinal stromal tumor. Ann Surg 245: 347-352, 2007.

59. Zalcberg JR, Verweij J, Casali GP, et al: Outcome of patients with advanced gastro-intestinal stromal tumours crossing over to a daily imatinib dose of $800 \mathrm{mg}$ after progression on $400 \mathrm{mg}$. Eur J Cancer 41: 1751-1757, 2005.

60. Demetri GD, van Oosterom AT, Garrett CR, et al: Efficacy and safety of sunitinib in patients with advanced gastrointestinal stromal tumour after failure of imatinib: a randomised controlled trial. Lancet 368: 1329-1338, 2006.

61. Bauer S, Yu LK, Demetri GD and Fletcher JA: Heat shock protein 90 inhibition in imatinib-resistant gatsrointestinal stromal tumor. Cancer Res 15: 9153-9161, 2006.

62. Bauer S, Duensing A, Demetri GD and Fletcher JA: KIT oncogenic signaling mechanisms in imatinib-resistant gastrointestinal stromal tumor: PI3-kinase/AKT is a crucial survival pathway. Oncogene 26: 7560-7568, 2007

63. Pantaleo MA, Fanti S, Nannini M, et al: What oncologists need and require from nuclear medicine. Eur J Nucl Med Mol Imaging 35: 1761-1765, 2008.

64. Grimpen F, Yip D, McArthur G, Waring P, et al: Resistance to imatinib, low-grade FDG-avidity on PET, and acquired KIT exon 17 mutation in gastrointestinal stromal tumour. Lancet Oncol 6: 724-727, 2006.

65. Stroobants S, Goeminne J, Seegers M, et al: 18FDG-positron emission tomography for the early prediction of response in advanced soft tissue sarcoma treated with imatinib mesylate (Glivec). Eur J Cancer 39: 2012-2020, 2003

66. Gayed I, Vu T, Iyer R, et al : The role of 18FDG-PET in staging and early prediction of response to therapy of recurrent gastrointestinal stromal tumours. J Nucl Med 45: 17-21, 2004.

67. Goerres GW, Stupp R, Barghouth G, et al: The value of PET, $\mathrm{CT}$, and in line PET/CT in patients with gastrointestinal stromal tumours: long-term outcome of treatment with imatinib mesylate. Eur J Nucl Med Mol Imaging 32: 153-162, 2005.

68. Van den Abbeele AD: The lessons of GIST-PET and PET/CT: a new paradigm for imaging. Oncologist 13 (Suppl. 2): S8-S13, 2008.

69. Barthel H, Cleij MC, Collingridge DR, et al: 3'-Deoxy-3'(18F)fluorothymidine as a new marker for monitoring tumor response to antiproliferative therapy in vivo with positron emission tomography. Cancer Res 63: 3791-3798, 2003.

70. Kil KE, Ding YS, Lin KS, et al: Synthesis and positron emission tomography studies of carbon-11-labeled imatinib (Gleevec). Nucl Med Biol 34: 153-163, 2007.

71. Wang H, Cai W, Chen K, et al: A new PET tracer specific for vascular endothelial growth factor receptor 2. Eur J Nucl Med Mol Ima 34: 2001-2010, 2007.

72. Dimitrakopoulou-Strauss A, Hohenberger P, Haberkorn U, et al: $68 \mathrm{Ga}$-labeled bombesin studies in patients with gastrointestinal stromal tumors: comparison with 18F-FDG. J Nucl Med 48: 1245-1250, 2007

73. Antonescu CR, Viale A, Sarran L, et al: Gene expression in gastrointestinal stromal tumours is distinguished by KIT genotype and anatomic site. Clin Cancer Res 10: 3282-3290, 2004.

74. Agaram NP, Besmer P, Wong GC, et al: Pathologic and molecular heterogeneity in imatinib-stable or imatinib-responsive gastrointestinal stromal tumours. Clin Cancer Res 13: 170-181, 2007.

75. Meza-Pezeda LA, Kresse SH, Brragan-Polonia AH, et al: Array comparative genomic hybridization reveals distinct DNA copy number differences between gastrointestinal stromal tumours and Leiomyosarcomas. Cancer Res 66: 8984-8993, 2006

76. Weir BA, Woo MS, Getz G, et al: Characterizing the cancer genome in lung adenocarcinoma. Nature 450: 893-898, 2007

77. Tarn C, Rink L, Merkel E, et al: Insulin-like growth factor 1 receptor is a potential therapeutic target for gastrointestinal stromal tumors. Proc Natl Acad Sci USA 24: 8387-8392, 2008. 